\title{
ELABORAÇÃO DE ZONEAMENTOS AGROPEDOCLIMÁTICOS POR GEOPROCESSAMENTO: SOJ A EM MUNICÍPIOS DO RIO GRANDE DO SUL ${ }^{(1)}$
}

\author{
W. CARVALHO J UNIOR ${ }^{(2)}$, C. S. CHAGAS $^{(2)}$, \\ N. R. PEREIRA ${ }^{(2)} \&$ J . C. M. STRAUCH ${ }^{(3)}$
}

\begin{abstract}
RESUMO
Os zoneamentos agropedoclimáticos fazem uso de banco de dados ambientais e apresentam uma classificação que integra diversas variáveis por meio das operações de geoprocessamento. Neste trabalho, foi realizado um estudo de caso da soja no Rio Grande do Sul. Os aplicativos PC-Arclnfo, Arcview e SGI VGA foram empregados para as operações de entrada, de geoprocessamento dos dados e de apresentação dos resultados alcançados. 0 geoprocessamento envolveu a reclassi ficação e cruzamentos dos planos de informação e operações com tabelas associadas aos planos de informação. As características dos solos, tais como: fertilidade natural, textura, relevo, profundidade efetiva, susceptibilidade à erosão, drenagem, pedregosidade/rochosidade e saturação por sódio, foram avaliadas para a cultura da soja em todos os componentes das unidades de mapeamento de solos do estado do Rio Grande do Sul, gerando um plano de informação de aptidão pedológica para essa cultura que, depois de cruzado com o de época de semeadura por municípios, gerou o plano de informação final do zoneamento agropedoclimático. A utilização desse método permiti u fazer uma avaliação: (a) de cada um dos componentes das unidades de mapeamento, e (b) dos resultados obtidos de acordo com a percentagem de ocorrência de cada componente dentro da unidade de mapeamento.
\end{abstract}

Termos de indexação: solos, banco de dados, SIG, interpretação.

(1) Recebido para publicação em fevereiro de 2001 e aprovado em fevereiro de 2003.

(2) Pesquisador da Embrapa Solos. Rua J ardim Botânico 1024. CEP 22460-000 Rio deJ aneiro(RJ ). E-mail: waldir@cnps.embrapa.br; cesar@cnps.embrapa.br; nilson@cnps.embrapa.br

(3) Pesquisadora, Escola Nacional de Ciências Estatísticas - ENCE. Rua André Cavalcanti 106, CEP 20231-050 Rio de J aneiro (RJ ). E-mail: juliast@ibge.gov.br 


\title{
SUMMARY: AGROPEDOCLIMATIC ZONING BY GEOPROCESSING: SOYBEAN IN MUNICIPALITIES OF RIO GRANDE DO SUL STATE
}

\begin{abstract}
Environmental databases and several variables based on geoprocessing operations are used for a classification of Agropedoclimatic Zoning. This paper presents a case study of soybean carried out in theState of Rio GrandedoSul. PC-Arcl nfo, Arcview, and SGI VGA softwares were used for data input, geoprocessing, and result presentation. Geoprocessing included thereclassification and overlay operations of information layers and their associated tables. Soil characteristics, such as fertility, texture, relief, effective depth, erosion suscepti bility, drainage, rocks, and sodium saturati on wereassessed for soybean culturefor all components of the soil map units of Rio Grande do Sul. An information layer for the pedologic aptitude of this culture was created and then overlaid with thesowing timelayer for municipal districts, generating thefinal information for Agropedoclimatic Zoning. This method allowed the evaluation of: (a) every single component of the map units, and (b) results obtained in agreement with the percentage of occurrence of each component within the map unit.
\end{abstract}

Index terms: soil, data base, SIG, interpretation.

\section{INTRODUÇÃO}

Os avanços tecnológicos da cartografia automatizada, dos sistemas de gerenciamento de banco de dados e do processamento digital deimagens, aliados ao desenvolvimento da computação, permitiram produzir um conjunto distinto de ferramentas para a captura automática de dados relacionados com superfície terrestre para o gerenciamento, análise e apresentação das informações geradas. A ligação técnica e conceitual do desenvol vimento destas ferramentas resultou no desenvolvimento de uma enorme variedade de métodos de processamento de dados geográficos. Este processamento tem sido chamado, na literatura nacional, de Geoprocessamento (Câmara \& Medeiros, 1996) e de Geomática (Ostensen, 1995; Clement et al., 1997). Tais termos têm sido usados para designar o campo da atividade que, sistematicamente, integra todos os meios usados para adquirir e gerenciar dados relacionados com a superfície terrestre e produzir informações geográficas usando os recursos da informática.

O geoprocessamento gerou uma variedade de ferramentas para atender às diversas necessidades dos usuários. Dentre elas, cabe destacar o Sistema de Informações Geográficas (SIG) que oferece capacidade dearmazenar os dados de diversas fontes, manipular, analisar e apresentar estas informações em um formato passível de ser compreendido pelo usuário (Souza et al., 1993). Um SIG tem como principal característica a indexação dos dados pela local ização como forma fundamental de organizá-los e manipulá-los, estabel ecendo a associação entre o dado e o local onde ocorre. Ele permite explorar a capacidade dos dados para gerar informações por mei o de análises espaciais interativas, que fornecem soluções rápidas e precisas para problemas relacionados com a distribuição, espacial etemporal, dos dados.

A utilização das técnicas de Geoprocessamento nos planejamentos da área ambiental tem aumentado significativamente nos últimos anos, uma vez que essas técnicas requerem análises e representações na forma de mapas para a comunicação de informações relacionadas com o meio ambiente. Isto pode ser observado nos últimos eventos nacionais, como, por exemplo, na XIII Reunião Brasileira de Manejoe Conservação do Solo e da Água (2000), no XXVI e no XXVII Congresso Brasileiro de Ciência do Solo $(1997,1999)$ e no GISBRASIL 2000 (2000), ocasião em que os trabalhos apresentados comprovaram o uso de técnicas de análises espaciais encontradas em ambientes SIG.

A realização de planejamentos ambientais confiáveis requer a análise de um grande volume de dados, que devem ser manuseados de forma integrada. Segundo Donzeli et al. (1992), o grande volume de dados e a sua dinâmica espaço-temporal são características deste enfoque e requerem uma col eta de dados que atendam àquelas exigências de forma ágil e de custo relativamente baixo. Nestas circunstâncias, as técnicas de geoprocessamento encontradas em ambientes de SIG constituem importantes ferramentas que podem ser utilizadas para atender a tais objetivos.

Para subsidiar a gestão ambiental no Brasil, algumas iniciativas no desenvolvimento de ferramentas e métodos de Geoprocessamento têm sido desenvolvidas, principalmente a partir da década de 80, quando foram iniciados os estudos de impacto ambiental (EIA). Dentreas iniciativas, cabe 
destacar os esforços do Instituto Nacional de Pesquisas Espaciais (INPE) e da Imagem, para o desenvolvimento doSPRING edoSGINGA (Câmara \& Medeiros, 1996), e do Instituto de Geografia/ UFRJ , para o desenvolvimento doSAGA (Xavier da Silva \& Souza, 1988). No âmbito internacional, destacam-seas iniciativas da Clark University, para o desenvolvimento do IDRISI, do International Institute for Aerospace Survey and Earth Sciences (ITC), para o desenvolvimento do ILWIS, e do Environmental Systems Research Institute, para o desenvolvimento do ARC/I nfo. Estes aplicativos vêm sendo utilizados em conjunto, procurando-se aproveitar suas peculiaridades, potencialidades e facilidades.

Para efetuar análises ambientais, visando ao sucesso de projetos de desenvol vimento sustentável, os levantamentos de recursos naturais são indispensáveis. Esses levantamentos estratificam o ambiente em unidades homogêneas e permitem enfocar suas limitações ecológicas, determinando seu potencial de uso (Carvalho J unior, 1996). No que concerneao levantamento de sol os, muitas agências internacionais como: a USDA Soil Conservation Service, a Canadian National and Provincial Agency e a Dutch Soil Survey Institute (atualmente parte doStaring I nstitute for I ntegrated Land, Water and Rural Survey) vêm usando os ambientes deSI G com suas técnicas para mapear e prover informações sobre os solos e outros parâmetros de interesse para diferentes tipos de usuários (Maguire et al., 1991).

O objetivo deste trabal ho foi aplicar o método de Zoneamento Agropedoclimático da cultura da soja no estado do Rio Grande do Sul, apresentando, por questões de espaço, os resultados obtidos para apenas três municípios.

\section{MATERIAL E MÉTODOS}

N este trabal ho, foram estabel ecidas duas metas: a primeira consistiu na construção da base de dados geográficos em ambiente de SIG e a segunda na elaboração do Zoneamento Agropedoclimático propriamente dito.

\section{Método utilizado na elaboração da base de dados geográficos}

A elaboração da base de dados geográficos, em ambiente de SI G quesuporta o desenvol vimento dos trabalhos do zoneamento, consistiu das seguintes etapas:

\section{Definição da base de dados geográficos}

Os trabalhos executados em ambiente SIG iniciaram-se pela definição das feições mínimas necessárias, com relação à base cartográfica, dos mapas temáticos eseus respectivos atributos. Assim, o banco de dados ficou constituído pelos seguintes planos deinformação: (a) da base cartográfica di gital, a saber: hidrografia, rodovia e divisão municipal; (b) de solos e (c) de aptidão climática, também denominado época de semeadura. Todos estes na escala de 1:1.000.000.

Com relação ao plano de informação de solos, foram definidos os atributos das unidades de mapeamento, estabelecidos no Mapa Exploratório dos Solos do estado do Rio Grande do Sul (IBGE \& EMBRAPA, 1991), que interferem diretamente na produção sustentada da cultura avaliada, que são: fertilidade natural do solo, textura, relevo, profundidade do solo, susceptibilidade à erosão, drenagem, pedregosidade/rochosidade e saturação por sódio. Destes atributos, a fertilidade e a susceptibilidade à erosão necessitaram de uma interpretação prévia, ou seja, para o estabelecimento das classes de fertilidade, foram utilizados os dados de capacidade de troca de cátions (Valor T), saturação por bases (Valor V) e saturação por alumínio, e para o estabelecimento das classes de suscepti bilidadeà erosão, utilizaram-se os dados de declividade, textura e profundidade do solo.

\section{Aquisição dos dados}

Os dados utilizados na elaboração do potencial pedoclimático do estado do Rio Grande do Sul para a cultura da soja foram extraídos de fontes secundárias, a saber: (a) Mapa Exploratório dos Solos do estado do Rio Grande do Sul (IBGE \& EMBRAPA, 1991) e (b) Zoneamento Agrícola do Ministério da Agricultura e do Abastecimento: Rio Grande do Sul: soja: safra 99/2000 (Brasil, 2000). Os requerimentos edáficos da cultura foram obtidos de consultas a especialistas da cultura.

\section{Construção da base de dados}

Neste trabalho, foram selecionados e utilizados os seguintes aplicativos: (1) ambiente SI G SGI NGA (I magem Geosistemas e Comércio, 1995), para digitalizar os mapas, visto que esteaplicativo fornece uma estrutura de fácil utilização para a entrada de dados no formato vetorial, bem como possibilita a transferência dos dados para outros aplicativos, além de ser todo em língua portuguesa; (2) PC - Arcl nfo (ESRI, 1994), para efetuar o processamento e as anál ises espaciais, uma vez que permiteo cruzamento de planos de informação no formato vetorial, e (3) ArcView (ESRI , 1994), para complementar a análise deresultados eelaborar as saídas gráficas na forma de mapas e tabelas.

Desta forma, a base cartográfica e os mapas temáticos foram digitalizados no ambiente SIG, enquanto os dados alfanuméricos foram digitados na forma de tabelas em planilhas el etrônicas.

A etapa de geocodificação, que trata da associação dos dados alfanuméricos às suas respectivas representações nos mapas temáticos, foi efetuada no ambiente SIG do aplicativo PC-ARC/I nfo. A pós esta etapa, as feições cartográficas dos planos de 
informação representam as características temáticas descritas pel os dados alfanuméricos. Para estabel ecer o relacionamento do plano de informações de solos com o atributo nome da unidade de mapeamento, procura-se associar tais informações com as tabelas que contêm os novos atributos das características das unidades de mapeamento. Dentre os atributos tratados, foram abordados, mais especificamente, os seguintes: fertilidade natural do solo, textura, relevo, profundidade do solo, susceptibilidade à erosão, drenagem, pedregosidade/rochosidade e saturação por sódio, para cada um dos componentes da unidade de mapeamento.

A operação deassociação dos dados al fanuméricos às suas feições espaciais foi realizada no módulo TABLES do PC - Arclnfo, equivalente ao módulo INFO do Ard nfo NT ou UNIX. O comando utilizado é o J OINITEM, que une dois arquivos de dados baseado em um item comum, que, no caso, é o nome da unidade de mapeamento. Desta maneira, materializa-se o banco de dados geográficos em ambienteSIG.

\section{Desenvolvimento de operações para o Zoneamento Agropedoclimático}

A partir da base de dados geográficos desenvolvida em ambiente SIG, são executados procedimentos computacionais para a seleção espacial, usando as características dos atributos, reclassificação, cruzamentos, análises de resultados (tabelas) e geração de saída gráfica (mapas). Para melhor compreensão, esta etapa será apresentada na seção a seguir.

\section{Método utilizado no Zoneamento Agropedo- climático}

O método adotado segue um raciocínio lógico de manipulação de informações georreferenciadas, tendo em vista o Zoneamento Agropedoclimático (Figura 1).

Cabe ressaltar que este trabal ho aplicou a lógica boleana na análise dos atributos que qualificam a apti dão agrícola dos sol os. Todavia, a mesma análise está sendo real izada com a lógica bayesiana ea lógica

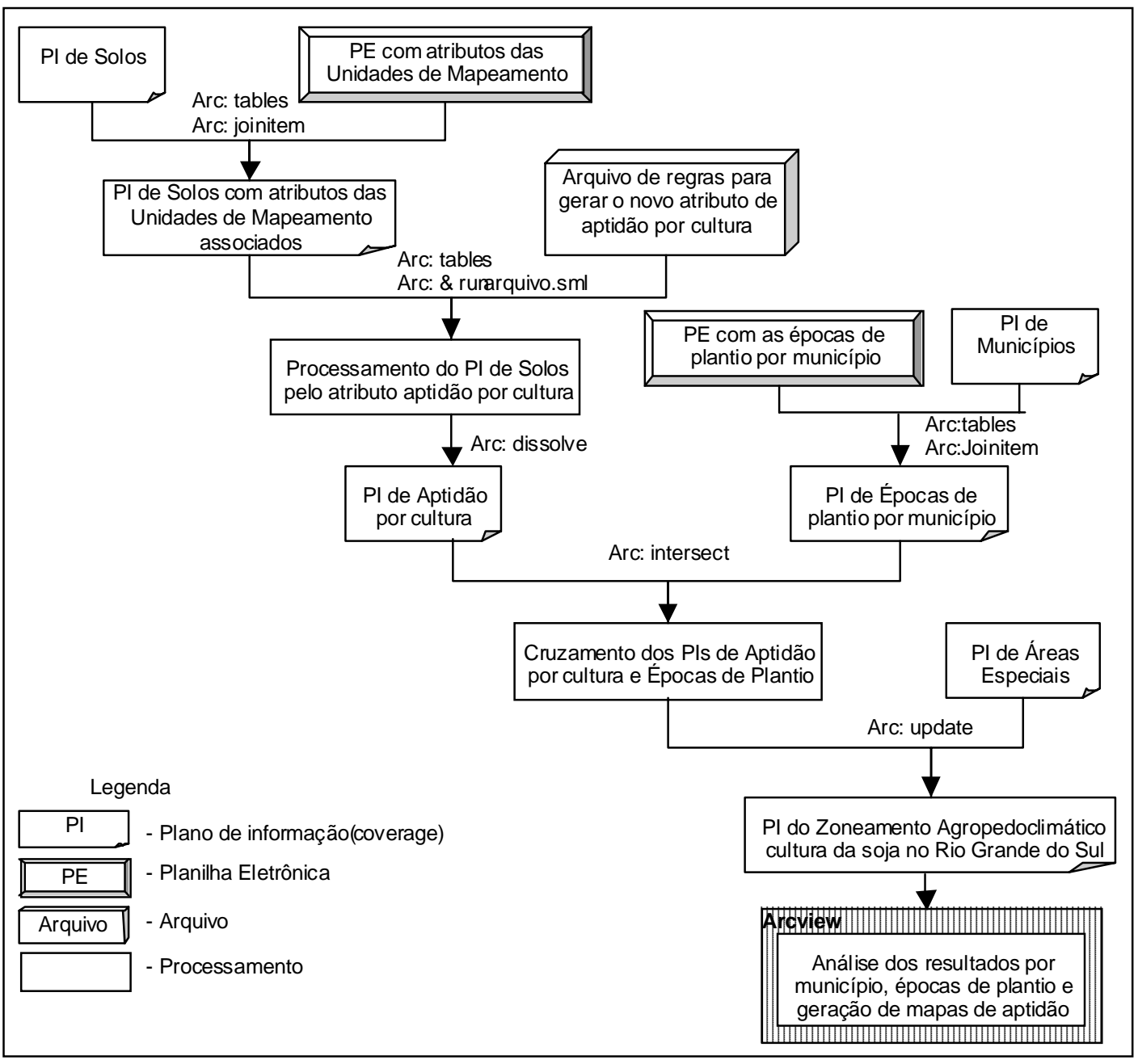

Figura 1. Fluxo de trabalho. 
fuzzy no mesmo ambienteSI G de forma a comparar os resultados al cançados.

Objetivando elaborar o mapa de aptidão dos sol os para determinada cultura (nestecasoa soja), écriado um arquivo deregras escrito em SML (Sample Macro Language). Esse arquivo descreve sel eções dentro da tabela associada ao plano de informação para gerar o atributo com a avaliação da aptidão. A aplicação das regras compreende um processo de seleções seqüenciais por características dos atributos da tabela associada ao plano de informação de solos de forma restritiva. No quadro 1, é apresentado um exemplo das características dos solos que determinam a classe de aptidão Boa para a cultura da soja, considerando a adoção de alto nível tecnológico.

$\mathrm{Na}$ figura 2, é ilustrado o arquivo de regras com as características apresentadas no quadro 1 e os respectivos comandos em SML, para selecionar as unidades de mapeamento que contenham as características de aptidão Boa. Como pode ser observado, o arquivo de regras consiste em um arquivo texto que é interpretado pelo módulo TABLES do PC-Arcl nfo.

Na figura 2, a linha 01 especifica a seleção de toda a tabela do plano de informação de solos (para o estado do Rio Grande do Sul). $\mathrm{Na}$ linha 02, começa a restrição da seleção para aquelas características de fertilidade consideradas e inerentes às unidades de mapeamento. Na linha 03, ocorreuma seleção, a partir da seleção anterior, das unidades de mapeamento que dispõem de novas condições que caracterizam o atributo textura, eassim por diante, até completar todos os requisitos necessários para que a unidade de mapeamento seja enquadrada na classe de aptidão pedológica Boa. Na última linha (linha n), o comando registra o nome da classe de aptidão no campo próprio daquelas unidades de mapeamento sel ecionadas. Assim, com este arquivo completo, com todas as classes de aptidão definidas (Boa, Regular el napta), basta executá-lo no módulo TABLES do PC - Arclnfo. Este procedimento é realizado para todos os componentes das unidades de mapeamento.

Dentre as vantagens da utilização do arquivo de regras em SML, destaca-se a facilidade de se realizarem ajustes e reinterpretações, pois basta alterar o arquivo de regras para as novas definições, além do fato de que todo o processamento fica documentado.

A partir deste processamento, obtêm-se o plano de informação de solos, que contém atributos associados, e o plano que foi criado pelo arquivo de regras, que contém as classes de aptidão dos solos. Este novo atributo criado será utilizado para processar a reclassificação do mapa de sol os e gerar o mapa de aptidão dos sol os para a cultura (soja). O comando DISSOLVE realiza esta operação, unindo polígonos adjacentes que tenham o mesmo val or do atributo considerado, gerando, assim, o mapa de aptidão dos solos para a cultura avaliada.

Concomitantemente à avaliação da aptidão dos solos, é elaborado o plano de informação de aptidão climática, que contém as épocas recomendadas de semeadura para os municípios do estado. Esteplano

\section{Quadro 1. Exemplo das características necessárias à definição das regras para a classe de aptidão Boa}

\begin{tabular}{ll}
\multicolumn{1}{c}{ Atributo } & \multicolumn{1}{c}{ Característica } \\
\hline Fertilidade & alta, média ou baixa \\
Textura & média, argilosa, muito argilosa, média/argilosa ou argilosa/muito argilosa \\
Relevo & plano ou suave ondulado \\
Profundidade efetiva & muito profunda ou profunda \\
Susceptibilidade à erosão & nula, nula a ligeira, ligeira ou ligeira a moderada \\
Drenagem & fortemente drenado, acentuadamente drenado ou bem drenado \\
Pedregosidade/rochosidade & ausente \\
Saturação por sódio & baixa \\
\hline
\end{tabular}

$$
\begin{aligned}
& \text { linha } 01 \text { - "select solos_rs.pat } \\
& \text { linha } 02 \text { - reselect fertilidade = 'alta' or 'média' or 'baixa' } \\
& \text { linha } 03 \text { - reselect textura = 'média' or 'argilosa' or 'muito argilosa' } \\
& \text { or 'média/argilosa' or 'argilosa/muito argilosa'...... } \\
& \text { linha n - move 'Boa' to aptidao_soja" }
\end{aligned}
$$

Figura 2. Exemplo de arquivo de regras em SML. 
de informação é gerado a partir da associação entre o plano de informação, que contém a malha municipal digital do estado (I BGE, 1999), ea tabela que contém as épocas de plantio por município (Brasil, 2000). Assim, este plano de informaçãoterá como atributos principais o nome do município, mesorregião, mi crorregião e época de semeadura.

Em seguida, o plano de informação de aptidão climática é então cruzado com o plano de informação da aptidão dos solos, gerando um novo plano, aqui denominado aptidão pedoclimática. Para efetuar esta operação, existem al gumas opções de comandos dentro do PC - Arcl nfo que podem ser utilizados, dependendo da situação e da necessidade. Dentre eles, podem ser citados o UNION, IDENTITY e o INTERSECT (Figura 3). Neste estudo de caso, foi utilizado o comando INTERSECT, que computa a interseção geométrica de dois planos de informação, conjugando seus atributos.

Neste estudo, foram identificadas também as "áreas especiais", compostas pelas unidades de conservação e áreas indígenas, visando à exclusão de suas potencialidades por serem regidas por legislação ambiental, não podendo constar de um zoneamento para fins agrícolas. Cabe ressaltar que só foram consideradas aquelas que apresentam dimensões superiores à área mínima mapeável na escala da base de dados. Nesta operação, foi utilizado o comando UPDATE do PC - Arcl nfo, queatualiza o mapa com os novos polígonos.

A partir deste momento, toda a operação é realizada dentro do ArcView e consiste na análise dos resultados ena geração de tabel as e mapas finais.

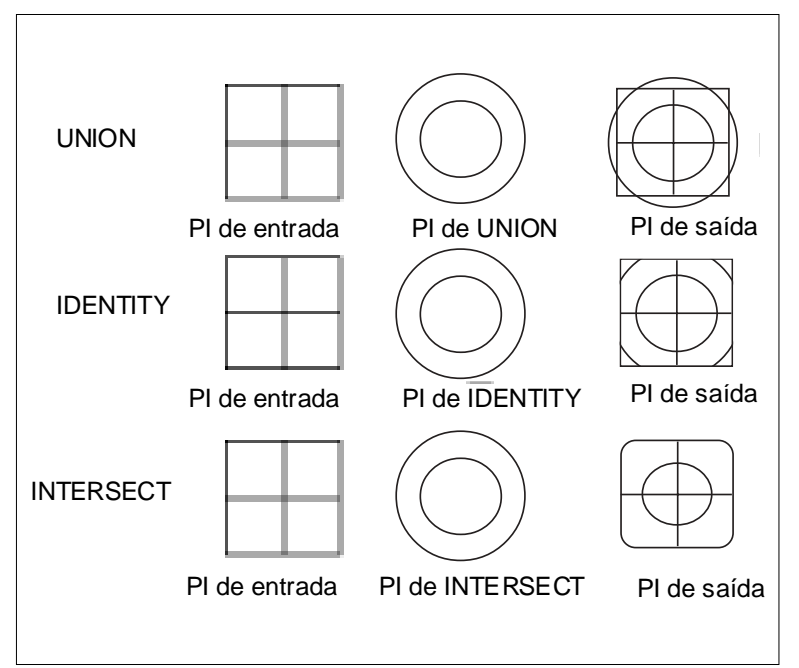

Figura 3. Operações espaciais de overlay no PC Arclnfo.

\section{RESULTADOS E DISCUSSÃO}

O primeiro resultado alcançado foi o banco de dados georreferenciados de solos, em que o mapa está associado a uma tabela com as características de seus atributos. Para ilustrar o trabalho, foram destacados os municípios de Ajuricaba, Condor e Nova Ramada, e os resultados são apresentados na figura 4 e no quadro 2.

Após a elaboração deste mapa de solos em ambiente SIG, o processamento utiliza o comando

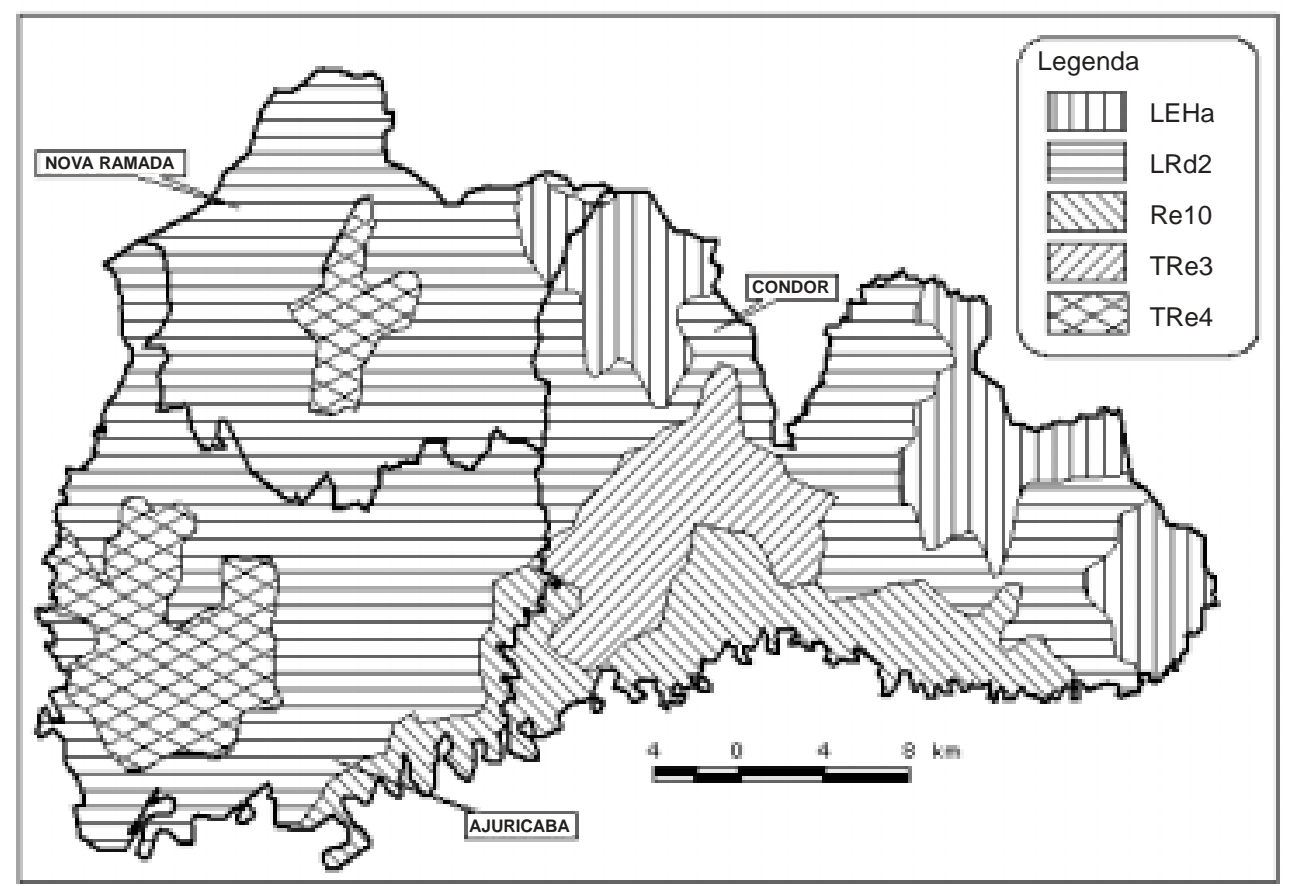

Figura 4. Distribuição espacial dos solos dos municípios de Ajuricaba, Condor e Nova Ramada. 
DISSOLVE a partir desta tabela de atributos, para gerar o plano de informação (PI) aptidão dos solos, O cruzamento deste com o PI de municípios e época de plantio gera o PI do Zoneamento Pedoclimático. Este PI, para o caso dos municípios de Ajuricaba, Condor e Nova Ramada, é apresentado na figura 5 e o quadro 3 apresenta a tabela da legenda.
O processo de contabilização de resultados consiste também na sel eção por atributos do plano de informação de aptidão pedoclimática. Neste plano de informação, é contabilizada a soma das áreas dos polígonos selecionados de acordo com os valores de seus atributos, no caso a aptidão dos solos, com referência ao percentual de ocorrência de cada um

Quadro 2. Exemplo da tabela associada ao mapa de solos com alguns de seus atributos

\begin{tabular}{|c|c|c|c|c|c|c|c|c|c|}
\hline UM & Textura & Relevo & Fertilidade & $\begin{array}{c}\text { Profundidade } \\
\text { efetiva }\end{array}$ & $\begin{array}{c}\text { Susceptibilidade } \\
\text { à erosão }\end{array}$ & Drenagem & Pedr/Roch & SatSodio & Aptidão \\
\hline LEHa & argilosa & suave ond. & baixa & m. profunda & nula a ligeira & acentuada & ausente & baixa & Boa \\
\hline LRd2 & m. argilosa & suave ond. & baixa & m. profunda & nula a ligeira & acentuada & ausente & baixa & Boa \\
\hline Rel0 & média casc. & ond. & alta & rasa & forte & boa & abundante & baixa & Inapta +Boa \\
\hline TRe3 & m. argilosa & suave ond. & média & profunda & ligeira & boa & ausente & baixa & Boa \\
\hline TRe4 & m. argilosa & ond. & média & profunda & ligeira a moderada & boa & ausente & baixa & Boa + Inapta \\
\hline
\end{tabular}

UM = símbolo da unidade de mapeamento, Pedr/Roch .= pedregosidade e, ou, rochosidade, SatSodio = saturação por sódio, $\mathrm{m} .=$ muito, ond. = ondulado e casc. = cascalhenta.

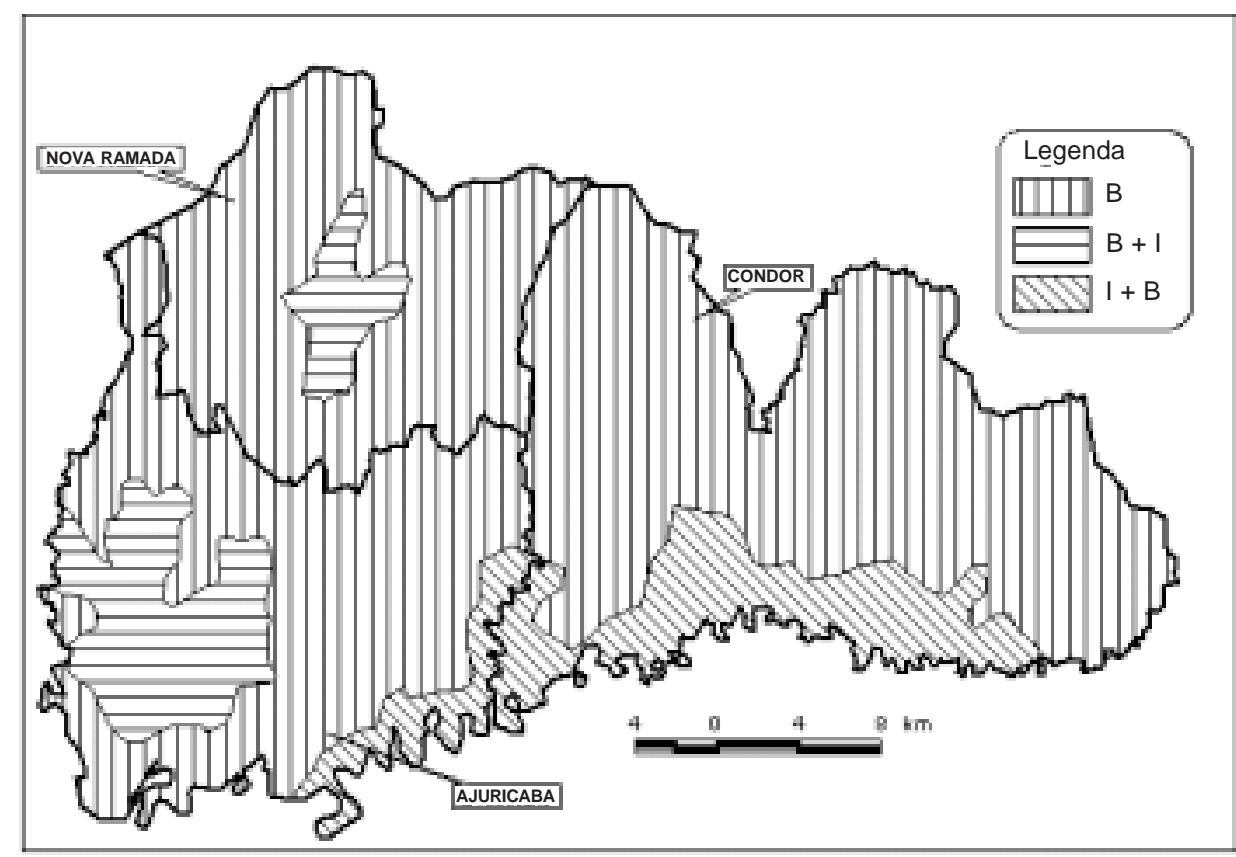

Figura 5. Distribuição espacial das classes de aptidão para os municípios estudados.

Quadro 3. Exemplo da tabela associada ao mapa do Zoneamento Pedoclimático com alguns de seus atributos

\begin{tabular}{|c|c|c|c|}
\hline Município & É poca de plantio & Área & Aptidão dos solos \\
\hline & & ha & \\
\hline $\begin{array}{l}\text { Ajuricaba } \\
\text { Ajuricaba } \\
\text { Ajuricaba }\end{array}$ & $\begin{array}{l}\text { 11/out. a 10/dez. } \\
\text { 11/out. a 10/dez. } \\
\text { 11/out. a 10/dez. }\end{array}$ & $\begin{array}{r}22.872 \\
7.710 \\
2.893\end{array}$ & $\begin{array}{l}\text { Boa } \\
\text { Boa + I napta } \\
\text { Inapta + Boa }\end{array}$ \\
\hline $\begin{array}{l}\text { Condor } \\
\text { Condor }\end{array}$ & $\begin{array}{l}\text { 11/out. a 10/dez. } \\
\text { 11/out. a 10/dez. }\end{array}$ & $\begin{array}{r}40.808 \\
9.463\end{array}$ & $\begin{array}{l}\text { Boa } \\
\text { Inapta + Boa }\end{array}$ \\
\hline $\begin{array}{l}\text { Nova Ramada } \\
\text { Nova Ramada }\end{array}$ & $\begin{array}{l}\text { 11/out. a 10/dez. } \\
\text { 11/out. a 10/dez. }\end{array}$ & $\begin{array}{r}24.510 \\
2.630\end{array}$ & $\begin{array}{l}\text { Boa } \\
\text { Boa + Inapta }\end{array}$ \\
\hline
\end{tabular}


Quadro 4. Resultados obtidos para alguns municípios do Rio Grande do Sul

\begin{tabular}{lcccc}
\hline \multirow{2}{*}{ Município } & Área do município & \multicolumn{3}{c}{ Classe de aptidão } \\
\cline { 3 - 5 } & & Preferencial & Tolerada & Não recomendada \\
\cline { 3 - 5 } & 33.476 & 28.656 & 0 & 4.820 \\
Ajuricaba & 46.487 & 40.808 & 0 & 5.687 \\
Condor & 25.563 & 24.510 & 0 & 1.052 \\
Nova Ramada & & & \\
\hline
\end{tabular}

Quadro 5. Distribuição das classes de aptidão pedoclimática para a soja no Rio Grande do Sul

\begin{tabular}{lcc}
\hline \multicolumn{1}{c}{ Classe de aptidão } & Área & $\begin{array}{c}\text { \% em relação } \\
\text { ao estado }\end{array}$ \\
\hline & $\mathrm{km}^{2}$ & \\
Preferencial & $77.066,11$ & 27,32 \\
Tolerada & $28.173,30$ & 9,99 \\
Não Recomendada & $161.665,43$ & 57,31 \\
Águas internas, áreas urbanas, etc & $15.157,16$ & 5,37 \\
\hline
\end{tabular}

dos componentes das unidades de mapeamento. Por exemplo, a unidade de mapeamento TRe4 é composta por Terra Roxa Estruturada (Nitossolo Vermelho) eSolos Litólicos (Neossol os Litólicos), que correspondem, respectivamente, a 60 e $40 \%$ de sua área total, e tem como aptidão do sol o a classe "Boa +I napta". Assim, a área relativa à aptidão de cada componente é cal culada segundo sua ocorrência na unidade de mapeamento. Neste caso, obtém-se 4.626 ha (60 \% de 7.710) de terras com aptidão Boa e 3.084 ha (40\% de 7.710$)$ de terras inaptas.

Este resultado é analisado para todo o estado, pela percentagem de cada classe de aptidão por município (Quadro 4).

Um resumo dos resultados al cançados para todo o estado pode ser observado no quadro 5, onde $37,31 \%$ das terras revelam aptidão para o plantio da soja, eqüivalente às classes (preferencial e tolerada), enquanto 57,31 \% não são recomendadas para o plantio (classe marginal enão recomendada).

\section{CONCLUSÕES}

1. Para executar Zoneamentos Agropedoclimáticos, deve ser trabalhado grande volume de dados relativos aos temas envolvidos, e o uso de SIG contribui para facilitar o manejo destes atributos espacialmente.

2. A utilização deSI G em trabal hos dezoneamento é recomendável, pois cria um banco de dados geor referenciados e permite atual izações e correções de modo rápido e bastante simples.

3. A utilização desse método permitiu avaliar as unidades de mapeamento para todos os seus componentes, bem como a percentagem deocorrência decada componente dentroda unidade de mapeamento.

\section{LITE RATURA CITADA}

BRASIL. Ministério da Agricultura e do Abastecimento. Coordenação Nacional do Zoneamento Agrícola. Zoneamento agrícola: safra 99/2000: Brasil: culturas algodão, arroz, feijão, maçã, milho, soja e trigo: estados RS, SC, PR, MG, SP, DF, GO, MT, MS, TO, AL, BA, CE, MA, PB, PE, PI, RN, SE. Brasília, 2000. Não paginado.

CÂMARA, G. \& MEDEIROS, J.S. Geoprocessamento para projetos ambientais. São J osé dos Campos, 1996. p39. (Relatório do INPE)

CARVALHO J UNIOR, W. Modelos de planejamento agrícola conservacionista com suporte de geoprocessamento. Estudo de caso nos municípios de Paty deAlferes e Miguel PereiraRJ . Rio de J aneiro, IGEO/UFRJ, 1996. p115. (Tese de Mestrado)

CLEMENT, G.; LAROUCHE, C.; GOUIN, D.; MORIN, P. \& KUCERA, H. OGDI: toward interoperability among geoespacial databases. SIGMOD Records, 26:18-23, 1997.

CONGRESSO BRASILEIRO DE CIÊNCIA DO SOLO, 26. Rio de J aneiro. 1997. Anais. Rio de J aneiro, Sociedade Brasileira de Ciência do Solo. 1997. CD-ROM

CONGRESSO BRASILEIRO DE CIÊNCIA DO SOLO, 27. Brasília. 2000, Anais. Brasília: Sociedade Brasileira de Ciência do Solo, 1999. CD-ROM.

DONZELI, P.L.; VALÉRIO FILHO, M.; PINTO, S.A.F.; NOGUEIRA, F.P.; ROTTA, C.L. \& LOMBARDI NETO, F. Técnicas de sensoriamento remoto aplicadas ao diagnóstico básico para planejamento e monitoramento de microbacias hidrográficas. Campinas, Instituto Agronômico de Campinas, 1992. p91-119. (Documentos IAC, 29)

ENVIRONMENTAL SYSTEM RESEARCH INSTITUTE. ESRI. PC ARC/INFO, Command references. New York, 1994. p.281.

GISBRASIL 2000, Salvador. Anais. Salvador, 2000. CD-ROM

INSTITUTO BRASILEIRO DE GEOGRAFIA E ESTATÍSTICA. Malha municipal digital do Brasil. Situação em 1997. Rio de J aneiro, 1999. CD-ROM 
INSTITUTO BRASILEIRO DE GEOGRAFIA E ESTATÍSTICA - IBGE/EMPRESA BRASILEIRA DE PESQUISA AGROPECUARIA - EMBRAPA. Serviço Nacional de Levantamento e Conservação de Sol os (Rio deJ aneiro, RJ ). Mapa Exploratório dos Solos do Estado do Rio Grande do Sul. In: CONGRESSO BRASILEIRO DE CIÊNCIA DO SOLO, 23., Porto Alegre 199I. Programa eresumos... [Porto Alegre]: Sociedade Brasileira de Ciência do Solo/ Universidade Federal do Rio Grande do Sul, 1991. p.264.

IMAGEM GEOSISTEMAS E COMÉRCIO. SGINGA, manual do usuário, versão 2.5. São J osé dos Campos, 1995.

MAGUIRE, D.J .; GOODCHILD, M.F. \& RHIND, D.W. Geographical Informations Systems. London, Longmam Scientific and Thecnical, 1991. 2v.
OSTENSEN, O. Mapping the future of geomatics, ISO/TC 211. Paris, Prentice Hall International, 1995. p.49. (Bulletin, 1)

REUNIÃO BRASILEIRA DE MANEJ O E CONSERVAÇÃO DO SOLO E DA ÁGUA, 13., 2000, IIhéus. 500 anos de uso do solo no Brasil. Anais. Ilhéus, 2000. CD-ROM

SOUZA, J .M.; FERRARI, R.; DUARTE, M. \& RAMIREZ, M. Uma arquitetura organizacional para Sistemas de informação Geográfica Orientada a objetos, In: CONFERÊNCIA LATINO AMERICANA SOBRE SISTEMAS DE INFORMAÇÕES GEOGRÁFICAS, São Paulo, 1993.

XAVIER DA SILVA, J . \& SOUZA, M.J.L. Análise ambiental. Rio de J aneiro, Universidade Federal do Rio de J aneiro, 1988. $198 p$ 
W. CARVALHO JUNIOR et al.

R. Bras. Ci. Solo, 27:379-387, 2003 